With compliments of the Author 


\title{
New Methodology for the Synthesis of 3-Substituted Coumarins via Palladium-Catalyzed Site-Selective Cross-Coupling Reactions
}

\author{
Sérgio Martins, ${ }^{a}$ Paula S. Branco, ${ }^{\mathrm{b}}$ María C. de la Torre, ${ }^{\mathrm{c}}$ Miguel A. Sierra, ${ }^{\mathrm{d}}$ António Pereira*a \\ a Centro de Química, Departamento de Química,, Universidade de Évora, 7000-671 Évora, Portugal \\ Fax +351(266)745303; E-mail: amlp@uevora.pt \\ b REQUIMTE, Departamento de Química, Faculdade de Ciências e Tecnologia, FCT, Universidade Nova de Lisboa, \\ 2829-516 Caparica, Portugal \\ c Instituto de Química Orgánica General, Consejo Superior de Investigaciones Científicas (CSIC), 28006 Madrid, Spain \\ d Departamento de Química Orgánica, Facultad de Química, Universidad Complutense, 28040 Madrid, Spain \\ Received 21 July 2010
}

\begin{abstract}
A particularly useful, easy, and concise synthesis of diversified 3-aryl coumarin was achieved using Heck coupling reactions between coumarin and aryliodides. The introduction of the aryl moiety occurred regioselective at the 3 -position of the heteroaromatic ring.
\end{abstract}

Key words: coumarin, Heck reaction, palladium, coupling reaction, 3-aryl coumarin

Coumarins, a common motif in a variety of naturally occurring compounds, have attracted intense interest in recent years due to their diverse pharmacological properties, namely as anticoagulant, antimicrobial, antibacterial, anticancer, anti-HIV, and antioxidant. ${ }^{1}$ Moreover, these series of compounds has outstanding optical properties as they constitute the largest class of laser dyes for the 'bluegreen' region ${ }^{2}$ and are widely used as emission layers in organic light-emitting diodes (OLED), ${ }^{3}$ optical brighteners, ${ }^{4}$ nonlinear optical chromophores, ${ }^{5}$ fluorescent whiteners, ${ }^{6}$ as well as fluorescent labels and probes for physiological measurement. ${ }^{7}$ Recently, coumarins have found wide applications in the labeling ${ }^{8}$ and caging. ${ }^{9}$ Another feature of the coumarin derivatives is that photophysical and spectroscopic properties can be readily modified by the introduction of substituents in the coumarin ring, giving themselves more flexibility to fit well into various applications. ${ }^{10}$ In particular, it seems that the presence of a heteroaryl moiety at the 3-position of the coumarinic system induces specific activities. ${ }^{11,12} \mathrm{Al}-$ though there are classical methods ${ }^{13}$ for the synthesis of a wide variety of substituted coumarins, these methods often require the use of strong acids and high temperatures. Much effort has been paid to the development of synthetic methods requiring the use of transition-metal catalyst. ${ }^{14,15}$ However, most of the methods rely on the construction of the coumarin skeleton starting from halogenated substrates such as iodophenols and iodoarenes. ${ }^{14,16}$ Methods relying on the derivatization of the coumarin ring are rare and limited to formerly substituted coumarins. ${ }^{17}$ Here we proposed to use palladium-catalyzed cross-coupling reac-

SYNLETT 2010, No. 19, pp 2918-2922

Advanced online publication: 03.11.2010

DOI: 10.1055/s-0030-1259014; Art ID: G23010ST

(c) Georg Thieme Verlag Stuttgart · New York tions which proceed stepwise and display site selectivity for the specific introduction of a heteroaryl moiety at the 3-position of the coumarin ring.

When stoichiometric amounts of coumarin (1) and iodobenzene (2a) were allowed to react using $\mathrm{Pd}(\mathrm{OAc})_{2}$ as catalyst, the insertion of the phenyl moiety occurred at the 3position of the coumarin ring in trace amounts (Table 1, entry 1).

Changing reaction conditions as the catalyst $\left[\mathrm{PdCl}_{2}\right.$, $\left.\mathrm{Pd}_{2}(\mathrm{dba})_{3}\right]$, the base $\left(\mathrm{Et}_{3} \mathrm{~N}, \mathrm{NaHCO}_{3}\right)$, the temperature, the solvent $\left(\mathrm{CH}_{2} \mathrm{Cl}_{2}, \mathrm{MeCN}\right)$, and the presence of a phasetransfer catalyst $\left(\mathrm{Bu}_{4} \mathrm{NBr}\right)$ revealed that the system coumarin (3.0 equiv), $\mathrm{PhI}$ (1.0 equiv), $\mathrm{Pd}\left(\mathrm{PPh}_{3}\right)_{4}(10 \mathrm{~mol} \%)$, $\mathrm{MeCO}_{2} \mathrm{Ag}$ (1.1 equiv), DMF, $80^{\circ} \mathrm{C}, 72 \mathrm{~h}$ was the most efficient to furnish the desired aryl substituted coumarin ${ }^{12}$ 3a (Scheme 1) in 52\% yield while 1-bromo-4-iodobenzene afforded the cross-coupled product $3 \mathbf{b}^{18,19}$ in $66 \%$ yield. (Table 1, entries 5 and 16). A slight excess of the coumarin was needed to ensure complete conversion of the aryl iodide. Competitive byproducts, as 1,1'-biphenyl or $4,4^{\prime}$ dibromo-1, $1^{\prime}$-biphenyl, were observed in particular on those reaction that presents a reduced yield of coumarin. These compounds result from competitive reactions between aryl iodide due to the poorer reactivity of the coumarin ring. Unexpectedly, 3-arylcoumarins 3 were the only monocoupled products formed. To demonstrate the generality of this strategy, the method was applied to other aryl iodides. ${ }^{20}$ Remarkably, electron-withdrawing as well as electron-donating substituents on the aryliodide are suitable partners in this process, affording the corresponding monosubstituted products in fair to good yields (Table 1, entries 17-21) although, electron-withdrawing groups gave best results as expected. Indeed, with electron-donating groups (Table 1, entries 17 and 21) the yield of the reaction decreased substantial which is related to the palladium insertion step.

Other catalyst were also tried as $\mathrm{Pd}\left(\mathrm{OCOCF}_{3}\right)_{2}, \mathrm{Pd}_{2}(\mathrm{dba})_{3}$, and $\mathrm{PdCl}_{2}$ (results not shown) but the results were disappointing. The introduction of aryl substituents at the 3-position of the coumarin scaffold extended the $\pi$-system of the parent chromophore which was observed by a batochromic shift of the absorbance maximum from $312 \mathrm{~nm}$ to around $325 \mathrm{~nm}$ The complete structural elucidation of 3 was accomplished using two-dimensional NMR spec- 


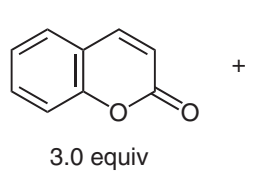

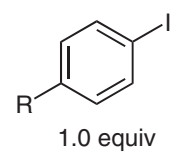

$2 \mathrm{a} \mathrm{R}=\mathrm{H} \quad 2 \mathrm{e} \mathrm{R}=\mathrm{CHO}$

$2 b \mathrm{~b}=\mathrm{Br} 2 \mathrm{ff} \mathrm{R}=\mathrm{NO}_{2}$

2c R = Et $2 \mathrm{gR}=\mathrm{OMe}$

2d $R=1$

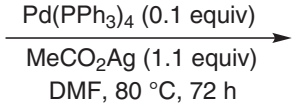

DMF, $80^{\circ} \mathrm{C}, 72 \mathrm{~h}$

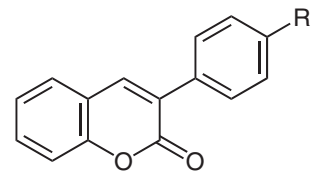

3a $\mathrm{R}=\mathrm{H} \quad 3$ e $\mathrm{R}=\mathrm{CHO}$

3c R = Et $3 \mathrm{~g} \mathrm{R}=\mathrm{OM}_{2}$

$3 \mathrm{~d} R=$

Scheme 1 Reagents and conditions for the synthesis of 3-phenyl coumarins

troscopy as the reported citation on literature for some of the coumarins described here lack strong spectroscopic evidence. Characteristic of these structures is the absence of the pair of doublets at $\delta=7.7$ and $6.4 \mathrm{ppm}$ of the H-4 and H-3 coumarin protons, respectively, and the appearance of a high deshielding singlet around $\delta=7.8-8.0 \mathrm{ppm}$ for $\mathrm{H}-4$ as a result of the absence of the coupling with the adjacent proton. Also, we observed the principal mode of fragmentation of 3-substituted coumarins which involves the elimination of carbon monoxide and the formation of the benzo $[b]$ furan radical cation. ${ }^{18}$ Regioselectivity is one of the major problems of Heck reactions and is related only to the coordination-insertion pathway. ${ }^{23}$ The coordination-insertion step of the Heck reaction can follow two reaction pathways: one involves coordination of the olefin via dissociation of a neutral ligand and another involves coordination of the olefin via dissociation of the anionic ligand.

Taking into consideration that the best results were observed when silver salts were used as bases (and as se-

Table 1 Yields and Reaction Conditions for the Synthesis of 3-Arylcoumarins

\begin{tabular}{|c|c|c|c|c|c|c|c|c|c|}
\hline Entry & 1 & & Catalyst (equiv) & Base (equiv) & Solvent & $\mathrm{Ph}_{3} \mathrm{P}$ & Temp $\left({ }^{\circ} \mathrm{C}\right)$ & Time (h) & Yield (\%) \\
\hline 1 & 1.0 & $2 a$ & $\mathrm{Pd}(\mathrm{OAc})_{2}(0.1)$ & $\mathrm{NaOAc}(2.0)$ & DMF & 0.4 & 60 & 24 & trace \\
\hline 2 & 3.0 & $2 \mathbf{a}$ & $\mathrm{Pd}(\mathrm{OAc})_{2}(0.1)$ & $\mathrm{NaOAc}(2.0)$ & DMF & 0.4 & 100 & 52 & 32 \\
\hline 3 & 3.0 & $\mathbf{2 a}$ & $\mathrm{Pd}(\mathrm{OAc})_{2}(0.1)$ & $\mathrm{AgOAc}(1.1)$ & DMF & 0.4 & 100 & 24 & 10 \\
\hline 4 & 3.0 & $2 \mathbf{a}$ & $\mathrm{Pd}\left(\mathrm{PPh}_{3}\right)_{4}(0.1)$ & $\mathrm{NaOAc}(4.0)$ & DMF & - & 100 & 24 & 35 \\
\hline 5 & 3.0 & $2 \mathbf{a}$ & $\operatorname{Pd}\left(\mathrm{PPh}_{3}\right)_{4}(0.1)$ & $\operatorname{AgOAc}(1.1)$ & DMF & - & 80 & 72 & 52 \\
\hline 6 & 1.1 & $2 b$ & $\mathrm{Pd}(\mathrm{OAc})_{2}(0.1)$ & $\mathrm{AgOAc}(1.1)$ & DMF & 0.4 & 100 & 24 & trace \\
\hline 7 & 3.0 & $2 \mathbf{b}$ & $\mathrm{Pd}(\mathrm{OAc})_{2}(0.1)$ & $\mathrm{NaOAc}(1.1)$ & DMF & 0.4 & 100 & 24 & trace \\
\hline 8 & 3.0 & $2 \mathbf{b}$ & $\mathrm{Pd}(\mathrm{OAc})_{2}(0.1)$ & $\operatorname{AgOAc}(1.1)$ & DMF & 0.4 & 100 & 48 & 15 \\
\hline 9 & 3.0 & $2 \mathrm{~b}$ & $\mathrm{Pd}(\mathrm{OAc})_{2}(0.1)$ & $\mathrm{AgOAc}(1.1)$ & DMF & 0.4 & 80 & 24 & 21 \\
\hline 10 & 3.0 & $2 b$ & $\mathrm{Pd}(\mathrm{OAc})_{2}(0.1)$ & $\mathrm{AgOAc}(1.1)$ & DMF & 2.0 & 100 & 24 & trace \\
\hline 11 & 3.0 & $2 b$ & $\mathrm{Pd}(\mathrm{OAc})_{2}(0.02)$ & $\mathrm{AgOAc}(1.1)$ & DMF & 0.08 & 100 & 24 & 35 \\
\hline 12 & 3.0 & $2 \mathbf{b}$ & $\mathrm{Pd}(\mathrm{OAc})_{2}(0.02)$ & $\mathrm{AgOAc}(1.1)$ & DMF & 0.08 & 80 & 48 & 13 \\
\hline 13 & 3.0 & $2 b$ & $\mathrm{Pd}(\mathrm{OAc})_{2}(0.02)$ & $\mathrm{AgOAc}(1.1)$ & DMF & 0.08 & 75 & 43 & 33 \\
\hline 14 & 3.0 & $2 \mathbf{b}$ & $\mathrm{Pd}(\mathrm{OAc})_{2}(0.1)$ & $\mathrm{AgOAc}(1.1)$ & DMF & - & 100 & 24 & trace \\
\hline 15 & 3.0 & $2 \mathbf{b}$ & $\mathrm{Pd}(\mathrm{OAc})_{2}(0.1)$ & $\mathrm{NaOAc}(1.1)$ & DMF & - & 100 & 24 & trace \\
\hline 16 & 3.0 & $2 \mathrm{~b}$ & $\mathrm{Pd}\left(\mathrm{PPh}_{3}\right)_{4}(0.1)$ & $\mathrm{AgOAc}(1.1)$ & DMF & - & 80 & 72 & 66 \\
\hline 17 & 3.0 & $2 c$ & $\mathrm{Pd}\left(\mathrm{PPh}_{3}\right)_{4}(0.1)$ & $\mathrm{AgOAc}(1.1)$ & DMF & - & 80 & 72 & 28 \\
\hline 18 & 3.0 & 2d & $\mathrm{Pd}\left(\mathrm{PPh}_{3}\right)_{4}(0.1)$ & $\mathrm{AgOAc}(1.1)$ & DMF & - & 80 & 72 & $54^{19,21}$ \\
\hline 19 & 3.0 & $2 e$ & $\mathrm{Pd}\left(\mathrm{PPh}_{3}\right)_{4}(0.1)$ & $\mathrm{AgOAc}(1.1)$ & DMF & - & 80 & 72 & 74 \\
\hline 20 & 3.0 & $2 f$ & $\mathrm{Pd}\left(\mathrm{PPh}_{3}\right)_{4}(0.1)$ & $\mathrm{AgOAc}(1.1)$ & DMF & - & 80 & 72 & $81^{22}$ \\
\hline 21 & 3.0 & $2 g$ & $\mathrm{Pd}\left(\mathrm{PPh}_{3}\right)_{4}(0.1)$ & $\mathrm{AgOAc}(1.1)$ & DMF & - & 80 & 72 & 12 \\
\hline
\end{tabular}




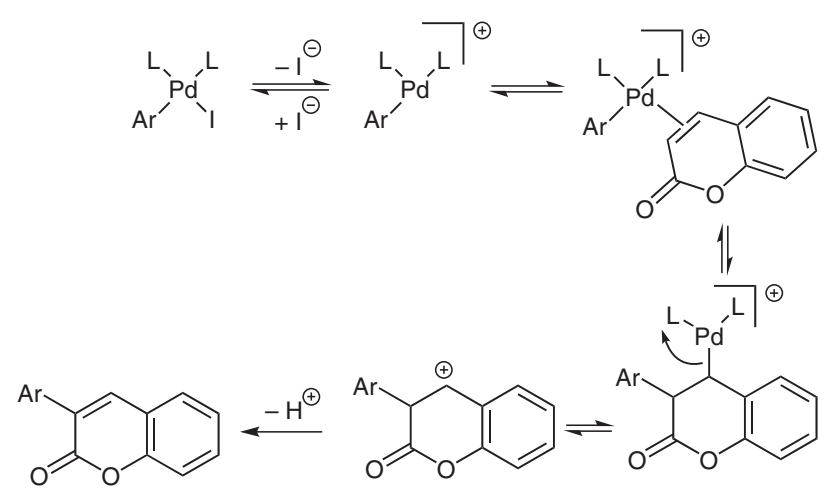

Scheme 2 Proposed mechanism for the synthesis of 3-aryl coumarins

questring agents for the halide anions), the last pathway mentioned above should be involved (Scheme 2). When the reaction proceeds via dissociation of the counterion as shown, electronic factors predominate. In fact, the coordination of the $\pi$-system with the cationic complex determines an increase of the polarization, and selective migration of the aryl moiety onto the carbon with the highest charge density is observed. With cyclic systems as is the case of the coumarin molecule, a syn- $\beta$-hydride elimination-dissociation process cannot be involved because there is no syn- $\beta$-hydrogen and rotation cannot occur. The efficiency of the process is related to the stabilized carbocation formed after dissociation of the catalytically active species of 14 -electron complex $\mathrm{L}_{2} \mathrm{P}(0)$. In view of our results we investigated the cross-coupling of methyl cinnamate derivatives and iodobenzene when the aforementioned reaction conditions were applied (Scheme 3 ). When the reaction was carried out with me- thyl trans-cinnamate derivatives $\mathbf{4 a}$ and $\mathbf{4 b}$ we observed the exclusive formation of the 3-phenyl monocoupled products $\mathbf{5} \mathbf{a}^{24}$ and $\mathbf{5 b}^{25}$ in $58 \%$ and $11 \%$ yield, respectively. The difference in the yield is probably related to stereo constraint due to the ortho-substitution. The spectroscopic data for $\mathbf{5 a}$ agree with those from literature. ${ }^{24}$ On the other hand, the reaction with the $Z$-derivative $\mathbf{6}$, a mimetic structure of the coumarin ring produces a mixture of 3and 2-phenyl monocoupled products $\mathbf{7}$ and $\mathbf{8}$ in the proportion of $1: 3$, respectively (obtained by ${ }^{1} \mathrm{H}$ NMR integration). ${ }^{25,26}$ The spectroscopic data ${ }^{26}$ reported for compound $\mathbf{8}$ do not match our result. Indeed the expected downfield signal for $\mathrm{H}-3$ (present in our case at $\delta=7.95 \mathrm{ppm}$ ) is absent on the report spectra. Our spectra is in harmony with that reported for the $E$-isomer of compound $\mathbf{8}$ that presents the H-3 signal at $\delta=8.16 \mathrm{ppm} .{ }^{27}$ The reported data for compounds $\mathbf{7}^{28}$ and $\mathbf{5} \mathbf{b}^{25}$ does not include NMR assignment which prevent us from a comparison with published data. Even so, the presence of a singlet near $\delta=6.2$ and $6.4 \mathrm{ppm}$, respectively, for the $\mathrm{H}-2$ proton is characteristic for these types of compounds. In view of these results the stereoelectronic properties of the alkene/ester group in the coumarin ring have a definitive role on the coordinationinsertion step of the group at C-3 position. Additional studies at the level of DFT (density functional theory) are currently under way.

The success of this method opens a new way to 3-substituted coumarins. Armed with this encouraging strategy, we briefly explored this synthetic methodology to the synthesis of diversified coumarins. For example a tandem Heck reaction of iodine derivative $\mathbf{3 d}$ with methyl acrylate, in the same conditions, provide the corresponding

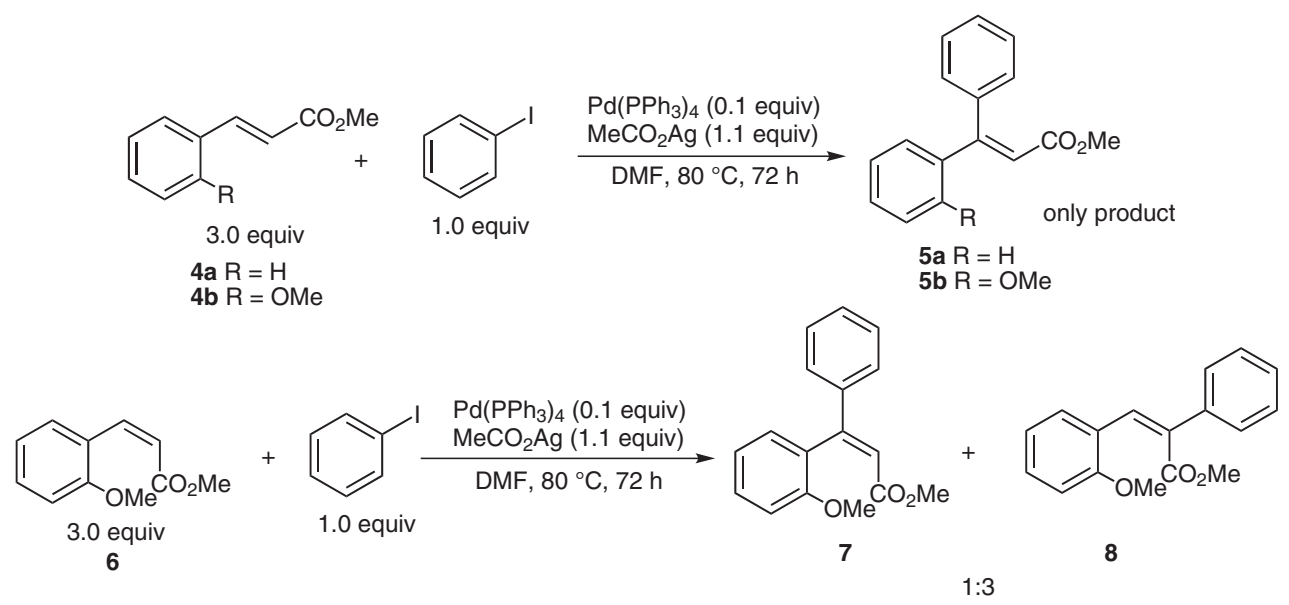

Scheme 3 Reaction of methyl cinnamate derivatives and iodobenzene<smiles>O=c1oc2ccccc2cc1-c1ccc(I)cc1</smiles>

$3 d$

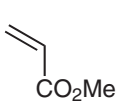

1.0 equiv
$\mathrm{Pd}\left(\mathrm{PPh}_{3}\right)_{4}$ (0.1 equiv) $\mathrm{MeCO}_{2} \mathrm{Ag}$ (1.1 equiv)

DMF, $80{ }^{\circ} \mathrm{C}, 72 \mathrm{~h}$ $96 \%$<smiles>CC(=O)C=Cc1ccc(-c2cc3ccccc3oc2=O)cc1</smiles>

9

Scheme 4 Synthesis of (E)-methyl-3-[4-(coumarin-3-yl)phenyl]acrylate (9) 
(E)-methyl-3-[4-(coumarin-3-yl)phenyl]acrylate 9 in $96 \%$ yield (Scheme 4$)$. This promising results display the potential of this strategy for numerous synthetic applications to diversified coumarins.

In summary, this new protocol considerably facilitates the concise synthesis of diversified 3-arylated coumarins by a Heck coupling reaction that proceeds essentially at C-3 rather than at $\mathrm{C}-4$ position on the coumarin ring. We also have demonstrated that sequential Heck reactions will enlarge the scope of this work with the synthesis of new and promising systems. To the best of our knowledge these types of reactions relying on the derivatization of the coumarin ring have not been widely published.

Supporting Information for this article is available online at http://www.thieme-connect.com/ejournals/toc/synlett.

\section{Acknowledgment}

We thank Fundação para a Ciência e Tecnologia (Lisbon, Portugal), PRAXIS program, for partial financial support FCOMP-01-0124FEDER-007448.

\section{References and Notes}

(1) (a) Hoult, J. R. S.; Paya, M. Gen. Pharmacol. 1996, 27, 713. (b) Kostova, I. Curr. Med. Chem. 2005, 5, 29. (c) Dayam, R.; Gundla, R.; Al-Mawsawi, L. Q.; Neamati, N. Med. Res. Rev. 2008, 28, 118. (d) Thuong, P. T.; Hung, T. M.; Ngoc, T. M.; Ha, D. T.; Min, B. S.; Kwack, S. J.; Kang, T. S.; Choi, J. S.; Bae, K. Phytother. Res. 2010, 24, 101. (e) Coumarins: Biology, Applications and Mode of Action; Wiley: Chichester, 1997.

(2) (a) Jones, G.; Jackson, W. R.; Choi, C.; Bergmark, W. R. J. Phys. Chem. 1985, 89, 294. (b) Koefod, R. S.; Mann, K. R. Inorg. Chem. 1989, 28, 2285. (c) Jagtap, A. R.; Satam, V. S.; Rajule, R. N.; Kanetkar, V. R. Dyes Pigm. 2009, 82, 84.

(3) (a) Lee, M. T.; Yen, C. K.; Yang, W. P.; Chen, H. H.; Liao, C. H.; Tsai, C. H.; Chen, C. H. Org. Lett. 2004, 6, 1241. (b) Swanson, S. A.; Wallraff, G. M.; Chen, J. P.; Zhang, W. J.; Bozano, L. D.; Carter, K. R.; Salem, J. R.; Villa, R.; Scott, J. C. Chem. Mat. 2003, 15, 2305. (c) Chang, C. H.; Cheng, H. C.; Lu, Y. J.; Tien, K. C.; Lin, H. W.; Lin, C. L.; Yang, C. J.; Wu, C. C. Org. Electron. 2010, 11, 247. (d) Yu, T. Z.; Zhang, P.; Zhao, Y. L.; Zhang, H.; Meng, J.; Fan, D. W. Org. Lett. 2009, 10, 653.

(4) (a) Dorlars, A.; Schellhammer, C. W.; Schroeder, J. Angew. Chem., Int. Ed. Engl. 1975, 14, 665. (b) Keskin, S. S.; Aslan, N.; Bayrakceken, N. Spectrochim. Acta, Part A 2009, $72,254$.

(5) (a) Moylan, C. R. J. Phys. Chem. 1994, 98, 13513.

(b) Mashraqui, S. H.; Mistry, H.; Sundaram, S.

J. Heterocycl. Chem. 2006, 43, 917. (c) Painelli, A.; Terenziani, F. Synth. Met. 2001, 124, 171.

(6) Wilze, K. A.; Johnson, A. K. Production, In Handbook of Detergents, Chemistry, Production, and Application of Fluorescent Whitening Agents, Part F; CRC Press, Taylor and Francis: Boca Raton / FL, 2007.

(7) (a) Kim, J. H.; Kim, H. J.; Kim, S. H.; Lee, J. H.; Do, J. H.; Kim, H. J.; Lee, J. H.; Kim, J. S. Tetrahedron Lett. 2009, 50, 5958. (b) Jung, H. S.; Kwon, P. S.; Lee, J. W.; Kim, J. I.; Hong, C. S.; Kim, J. W.; Yan, S. H.; Lee, J. Y.; Lee, J. H.;
Joo, T.; Kim, J. S. J. Am. Chem. Soc. 2009, 131, 2008. (c) Sheng, R. L.; Wang, P. F.; Gao, Y. H.; Wu, Y.; Liu, W. M.; Ma, J. J.; Li, H. P.; Wu, S. K. Org. Lett. 2008, 10, 5015. (d) Kim, H. J.; Park, J. E.; Choi, M. G.; Ahn, S.; Chang, S. K. Dyes Pigm. 2010, 84, 54. (e) Lin, W. Y.; Yuan, L.; Cao, X. W.; Tan, W.; Feng, Y. M. Eur. J. Org. Chem. 2008, 4981.

(8) (a) Goddard, J.-P.; Reymond, J.-L. Trends Biotechnol. 2004, 22, 363. (b) Heiner, S.; Detert, H.; Kuhn, A.; Kunz, H. Bioorg. Med. Chem. 2006, 14, 6149.

(9) (a) Mayer, G.; Heckel, A. Angew. Chem. Int. Ed. 2006, 45, 4900. (b) Geißler, D.; Antonenko, Y. N.; Schmidt, R.; Keller, S.; Krylova, O. O.; Wiesner, B.; Bendig, J.; Pohl, P.; Hagen, V. Angew. Chem. Int. Ed. 2005, 44, 1195.

(10) (a) Mizukami, S.; Okada, S.; Kimura, S.; Kikuchi, K. Inorg. Chem. 2009, 48, 7630. (b) Wagner, B. D. Molecules 2009, 14, 210. (c) Hara, K.; Kurashige, M.; Dan-oh, Y.; Kasada, C.; Shinpo, A.; Suga, S.; Sayama, K.; Arakawa, H. New J. Chem. 2003, 27, 783. (d) Camur, M.; Bulut, M.; Kandaz, M.; Guney, O. Supramol. Chem. 2009, 21, 624.

(11) (a) Matos, M. J.; Vina, D.; Picciau, C.; Orallo, F.; Santana, L.; Uriarte, E. Bioorg. Med. Chem. Lett. 2009, 19, 5053. (b) Matos, M. J.; Vina, D.; Quezada, E.; Picciau, C.; Delogu, G.; Orallo, F.; Santana, L.; Uriarte, E. Bioorg. Med. Chem. Lett. 2009, 19, 3268. (c) Kabeya, L. M.; da Silva, C.; Kanashiro, A.; Campos, J. M.; Azzolini, A.; Polizello, A. C. M.; Pupo, M. T.; Lucisano-Valim, Y. M. Eur. J. Med. Chem. 2008, 43, 996.

(12) Kabeya, L. M.; de Marchi, A. A.; Kanashiro, A.; Lopes, N. P.; da Silva, C.; Pupo, M. T.; Lucisano-Valima, Y. M. Bioorg. Med. Chem. 2007, 15, 1516.

(13) (a) Pechmann, V. H. D. C. Ber. Dtsch. Chem. Ges. 1884, 17, 929. (b) Perkin, W. H. J. Chem. Soc. 1875, 28, 10. (c) Johnson, J. R. Org. React. 1942, 1, 210. (d) Cairns, N.; Harwood, L. M.; Astles, D. P. J. Chem. Soc., Perkin Trans. 1 1994, 3101. (e) Jones, G. Org. React. 1967, 15, 204. (f) Brufola, G.; Fringuelli, F.; Piermatti, O.; Pizzo, F. Heterocycles 1996, 43, 1257. (g) Shirner, R. L. Org. React. 1942, 1, 1. (h) Narasimhan, N. S.; Mali, R. S.; Barve, M. V. Synthesis 1979, 906. (i) Yavari, I.; Hekmat-Shoar, R.; Zonouzi, A. Tetrahedron Lett. 1998, 39, 2391.

(14) Oyamada, J.; Kitamura, T. Tetrahedron 2006, 62, 6918.

(15) Cacchi, S. Pure Appl. Chem. 1996, 68, 45.

(16) (a) Kadnikov, D. V.; Larock, R. C. Org. Lett. 2000, 2, 3643. (b) Aoki, S.; Amamoto, C.; Oyamada, J.; Kitamura, T. Tetrahedron 2005, 61, 9291. (c) Kadnikov, D. V.; Larock, R. C. J. Org. Chem. 2003, 68, 9423.

(17) (a) Das, A. R.; Medda, A.; Singha, R. Tetrahedron Lett. 2010, 51, 1099. (b) Reddy, C. R.; Srikanth, B.; Rao, N. N.; Shin, D. S. Tetrahedron 2008, 64, 11666. (c) Zhang, L.; Meng, T.; Fan, R.; Wu, J. J. Org. Chem. 2007, 72, 7279.

(18) Vernin, G.; Coen, S.; Metzger, J.; Parkanyi, C. J. Heterocycl. Chem. 1979, 16, 97.

(19) Buuhoi, N. P.; Hoan, N.; Khenissi, M. R. J. Chem. Soc. 1951, 2307.

(20) General Procedure for the Pd-Catalyzed Coupling of Coumarin with Aryliodides

Under a nitrogen atmosphere, a mixture of coumarin (146 $\mathrm{mg}, 1.0 \mathrm{mmol}, 3.0$ equiv), aryl iodide (1.0 equiv), $\mathrm{Pd}\left(\mathrm{PPh}_{3}\right)_{4}$ (10 mol\%), and $\mathrm{MeCO}_{2} \mathrm{Ag}$ (1.1 equiv) in DMF $(2.0 \mathrm{~mL})$ was stirred at $80{ }^{\circ} \mathrm{C}$ for a period of $72 \mathrm{~h}$. The reaction mixture was diluted with EtOAc and washed with $\mathrm{H}_{2} \mathrm{O}$. The organic layer was dried $\left(\mathrm{Na}_{2} \mathrm{SO}_{4}\right)$, filtered, and concentrated under vacuum. The residue was purified by flash column chromatography on silica gel (230-400 mesh; hexane- $\mathrm{CH}_{2} \mathrm{Cl}_{2}$ gradient).

3-(4-Bromophenyl)coumarin (3b)

${ }^{1} \mathrm{H}$ NMR $\left(400 \mathrm{MHz}, \mathrm{CDCl}_{3}\right): \delta=7.31(1 \mathrm{H}, \mathrm{dd}, J=7.5,7.4$ 
$\mathrm{Hz}, \mathrm{H}-6), 7.37(1 \mathrm{H}, \mathrm{d}, J=8.5 \mathrm{~Hz}, \mathrm{H}-8), 7.53-7.61(6 \mathrm{H}, \mathrm{m}$, H-5, H-7, H-2', H-3', H-5', H-6'), $7.82(1 \mathrm{H}, \mathrm{s}, \mathrm{H}-4) .{ }^{13} \mathrm{C}$ $\operatorname{NMR}\left(100 \mathrm{MHz}, \mathrm{CDCl}_{3}\right): \delta=116.6(\mathrm{C}-8), 119.5(\mathrm{C}-4 \mathrm{a})$, $123.2\left(\mathrm{C}-4^{\prime}\right), 124.6(\mathrm{C}-6), 127.2$ (C-3), $128.0(\mathrm{C}-5), 130.1$ (C-2', C-6'), 131.7 (C-7, C-3', C-5'), 133.6 (C-1'), 139.9 (C-4), $153.6(\mathrm{C}-8 \mathrm{a}), 160.3(\mathrm{C}-2)$. MS $\left(\mathrm{EI}^{+}\right): \mathrm{m} / z(\%)=299$ $\left[\mathrm{M}^{79} \mathrm{Br}-\mathrm{H}\right]^{+}(100), 273\left[\mathrm{M}^{81} \mathrm{Br}-\mathrm{CO}\right]^{+}(71)$. HRMS $\left(\mathrm{EI}^{+}\right)$: $\mathrm{m} / \mathrm{z}$ calcd for $\mathrm{C}_{15} \mathrm{H}_{9} \mathrm{BrO}_{2}[\mathrm{M}]^{+}:$299.9786; found: 299.9790 . 3-(4-Ethylphenyl)coumarin (3c)

${ }^{1} \mathrm{H} \mathrm{NMR}\left(400 \mathrm{MHz}, \mathrm{CDCl}_{3}\right): \delta=1.27(3 \mathrm{H}, \mathrm{t}, J=7.6 \mathrm{~Hz}$, $\left.\mathrm{CH}_{2} \mathrm{CH}_{3}\right), 2.70\left(2 \mathrm{H}, \mathrm{q}, J=7.6 \mathrm{~Hz}, \mathrm{CH}_{2} \mathrm{CH}_{3}\right), 7.29(2 \mathrm{H}, \mathrm{d}$, $\left.J=8.2 \mathrm{~Hz}, \mathrm{H}-3^{\prime}, \mathrm{H}-5^{\prime}\right), 7.30(1 \mathrm{H}, \mathrm{dd}, J=7.7,7.7 \mathrm{~Hz}, \mathrm{H}-6)$, $7.37(1 \mathrm{H}, \mathrm{d}, J=8.0 \mathrm{~Hz}, \mathrm{H}-8), 7.52(1 \mathrm{H}, \mathrm{dd}, J=8.0,7.7 \mathrm{~Hz}$, $\mathrm{H}-7), 7.54(1 \mathrm{H}, \mathrm{d}, J=7.7 \mathrm{~Hz}, \mathrm{H}-5), 7.63(2 \mathrm{H}, \mathrm{d}, J=8.2 \mathrm{~Hz}$, $\left.\mathrm{H}-2^{\prime}, \mathrm{H}-6^{\prime}\right), 7.80(1 \mathrm{H}, \mathrm{s}, \mathrm{H}-4) .{ }^{13} \mathrm{C} \mathrm{NMR}\left(100 \mathrm{MHz}, \mathrm{CDCl}_{3}\right)$ : $\delta=15.5\left(\mathrm{CH}_{2} \mathrm{CH}_{3}\right), 28.7\left(\mathrm{CH}_{2} \mathrm{CH}_{3}\right), 116.4(\mathrm{C}-8), 119.8(\mathrm{C}-$ 4a), 124.4 (C-6), 127.8 (C-5), 128.0 (C-3', C-5'), 128.5 (C-3, C-2', C-6'), 131.2 (C-7), 132.0 (C-1'), 139.2 (C-4), 145.2 (C$\left.4^{\prime}\right), 153.4(\mathrm{C}-8 \mathrm{a}), 160.7(\mathrm{C}-2)$. MS $\left(\mathrm{EI}^{+}\right): \mathrm{m} / z(\%)=250[\mathrm{M}]^{+}$ (99.5), $235\left[\mathrm{M}-\mathrm{CH}_{3}\right]^{+}(100), 222[\mathrm{M}-\mathrm{CO}]^{+}$(17.87), 207 $\left[\mathrm{M}-\mathrm{CO}_{2}+\mathrm{H}\right]^{+}(77)$. HRMS $\left(\mathrm{EI}^{+}\right): \mathrm{m} / z$ calcd for $\mathrm{C}_{17} \mathrm{H}_{14} \mathrm{O}_{2}$ $[\mathrm{M}]^{+}:$250.0994; found: 250.0993 .

3-(4-Iodophenyl)coumarin (3d)

${ }^{1} \mathrm{H}$ NMR $\left(400 \mathrm{MHz}, \mathrm{CDCl}_{3}\right): \delta=7.31(1 \mathrm{H}, \mathrm{dd}, J=7.1,7.1$ $\mathrm{Hz}, \mathrm{H}-6), 7.37$ (1 H, d, $J=8.2 \mathrm{~Hz}, \mathrm{H}-8), 7.46(2 \mathrm{H}, \mathrm{d}, J=7.0$ $\left.\mathrm{Hz}, \mathrm{H}-2^{\prime}, \mathrm{H}-6^{\prime}\right), 7.53-7.56(2 \mathrm{H}, \mathrm{m}, \mathrm{H}-5, \mathrm{H}-7), 7.79(2 \mathrm{H}, \mathrm{d}$, $\left.J=7.0 \mathrm{~Hz}, \mathrm{H}-3^{\prime}, \mathrm{H}-5^{\prime}\right), 7.82(1 \mathrm{H}, \mathrm{s}, \mathrm{H}-4) .{ }^{13} \mathrm{C}$ NMR $(100$ $\left.\mathrm{MHz}, \mathrm{CDCl}_{3}\right): \delta=95.0\left(\mathrm{C}-4^{\prime}\right), 116.5(\mathrm{C}-8), 119.5(\mathrm{C}-4 \mathrm{a})$, 124.6 (C-6), 127.3 (C-3), 128.0 (C-5), 130.2 (C-2', C-6'), 131.7 (C-7), 134.2 (C-1'), 137.6 (C-3', C-5'), 139.9 (C-4), $153.6(\mathrm{C}-8 \mathrm{a}), 160.2(\mathrm{C}-2)$. MS $\left(\mathrm{EI}^{+}\right): \mathrm{m} / z(\%)=347$ $[\mathrm{M}]^{+}(61), 57\left[\mathrm{C}_{2} \mathrm{HO}_{2}\right](100)$. HRMS $\left(\mathrm{EI}^{+}\right): \mathrm{m} / z$ calcd for $\mathrm{C}_{15} \mathrm{H}_{9} \mathrm{IO}_{2}[\mathrm{M}]^{+}:$347.9647; found: 347.9645 .

\section{4-(Coumarin-3-yl)benzaldehyde (3e)}

${ }^{1} \mathrm{H}$ NMR $\left[400 \mathrm{MHz}, \mathrm{CO}\left(\mathrm{CD}_{3}\right)_{2}\right]: \delta=7.40(2 \mathrm{H}, \mathrm{m}, \mathrm{H}-6, \mathrm{H}-$ 8), $7.67(1 \mathrm{H}, \mathrm{dd}, J=7.2,6.8 \mathrm{~Hz}, \mathrm{H}-7), 7.80(1 \mathrm{H}, \mathrm{d}, J=6.8$ $\mathrm{Hz}, \mathrm{H}-5), 8.02$ (4 H, br s, H-2', H-3', H-5', H-6'), 8.31 (1 H, s, H-4), $10.10(1 \mathrm{H}, \mathrm{s}, \mathrm{CHO}) .{ }^{13} \mathrm{C}$ NMR [100 MHz, $\left.\mathrm{CO}\left(\mathrm{CD}_{3}\right)_{2}\right]: \delta=116.9(\mathrm{C}-8), 120.5(\mathrm{C}-4 \mathrm{a}), 125.5(\mathrm{C}-6)$, 127.5 (C-3), 129.7 (C-5), 130.1 (C-2', C-6'), 130.2 (C-3', C-5'), 133.0 (C-7), 137.4 (C-4'), 141.8 (C-1'), 142.4 (C-4), 154.8 (C-8a), 160.2 (C-2), 192.5 (CHO). MS (EI $\left.{ }^{+}\right): \mathrm{m} / z(\%)$ $249[\mathrm{M}-\mathrm{H}]^{+}$(73.71), $221[\mathrm{M}-\mathrm{CO}]^{+}$(14.6), $220[\mathrm{M}-$ $\mathrm{CHO}^{+}(17.9), 57\left[\mathrm{C}_{2} \mathrm{HO}_{2}\right](100)$. HRMS $\left(\mathrm{EI}^{+}\right): \mathrm{m} / z$ calcd for $\mathrm{C}_{16} \mathrm{H}_{10} \mathrm{O}_{3}[\mathrm{M}]^{+}$250.0630; found: 250.0632 .

3-(4-Nitrophenyl)coumarin (3f)

${ }^{1} \mathrm{H}$ NMR $\left(400 \mathrm{MHz}, \mathrm{CDCl}_{3}\right): \delta=7.36(1 \mathrm{H}, \mathrm{dd}, J=7.6,6.8$ $\mathrm{Hz}, \mathrm{H}-6), 7.41(1 \mathrm{H}, \mathrm{d}, J=8.2 \mathrm{~Hz}, \mathrm{H}-8), 7.60(1 \mathrm{H}, \mathrm{d}, J=7.6$ $\mathrm{Hz}, \mathrm{H}-5), 7.61(1 \mathrm{H}, \mathrm{dd}, J=8.2,6.8 \mathrm{~Hz}, \mathrm{H}-7), 7.92(2 \mathrm{H}, \mathrm{d}$,
$\left.J=8.8 \mathrm{~Hz}, \mathrm{H}-2^{\prime}, \mathrm{H}-6^{\prime}\right), 7.95(1 \mathrm{H}, \mathrm{s}, \mathrm{H}-4), 8.33(2 \mathrm{H}, \mathrm{d}$, $\left.J=8.8 \mathrm{~Hz}, \mathrm{H}-3^{\prime}, \mathrm{H}-5^{\prime}\right) .{ }^{13} \mathrm{C} \mathrm{NMR}\left(100 \mathrm{MHz}, \mathrm{CDCl}_{3}\right): \delta=$ 116.7 (C-8), 119.1 (C-4a), 123.7 (C-3', C-5'), 124.9 (C-6), 126.1 (C-3), 128.4 (C-5), 129.5 (C-2', C-6'), 132.6 (C-7), 141.0 (C-1'), 141.6 (C-4), 147.8 (C-4'), 153.9 (C-8a), 159.8 $(\mathrm{C}-2)$. MS $\left(\mathrm{EI}^{+}\right): \mathrm{m} / \mathrm{z}(\%)=267[\mathrm{M}]^{+}(100), 239[\mathrm{M}-\mathrm{CO}]^{+}$ (17.4). HRMS (EI+): $m / z$ calcd for $\mathrm{C}_{15} \mathrm{H}_{9} \mathrm{NO}_{4}[\mathrm{M}]^{+}$: 267.0532; found: 267.0533 .

3-(4-Methoxyphenyl)coumarin (3g)

${ }^{1} \mathrm{H}$ NMR [400 MHz, $\left.\mathrm{CO}\left(\mathrm{CD}_{3}\right)_{2}\right]: \delta=3.84\left(3 \mathrm{H}, \mathrm{s}, \mathrm{OCH}_{3}\right)$, $7.01\left(2 \mathrm{H}, \mathrm{d}, J=8.0 \mathrm{~Hz}, \mathrm{H}-3^{\prime}, \mathrm{H}-5^{\prime}\right), 7.36(2 \mathrm{H}, \mathrm{m}, \mathrm{H}-6, \mathrm{H}-$ 8), $7.59(1 \mathrm{H}, \mathrm{dd}, J=7.8,7.5 \mathrm{~Hz}, \mathrm{H}-7), 7.75(3 \mathrm{H}, \mathrm{m}, \mathrm{H}-5$, $\left.\mathrm{H}-2^{\prime}, \mathrm{H}-6^{\prime}\right), 8.07$ (1 H, s, H-4). ${ }^{13} \mathrm{C}$ NMR [100 MHz, $\left.\mathrm{CO}\left(\mathrm{CD}_{3}\right)_{2}\right]: \delta=55.6\left(\mathrm{OCH}_{3}\right), 114.5\left(\mathrm{C}-3^{\prime}, \mathrm{C}-5^{\prime}\right), 116.7(\mathrm{C}-$ 8), 120.9 (C-4a), 126.0 (C-6, C-3), 129.1 (C-5, C-1'), 130.7 (C-2', C-6'), 131.9 (C-7), 139.4 (C-4), 154.0 (C-8a), 160.5 $\left(\mathrm{C}-4^{\prime}\right), 161.1(\mathrm{C}-2)$. MS $\left(\mathrm{EI}^{+}\right): \mathrm{m} / z(\%)=252[\mathrm{M}]^{+}(100), 209$ $\left[\mathrm{M}-\mathrm{CO}+\mathrm{CH}_{3}\right]^{+}$(53.2). HRMS $\left(\mathrm{EI}^{+}\right): \mathrm{m} / z$ calcd for $\mathrm{C}_{16} \mathrm{H}_{12} \mathrm{O}_{3}[\mathrm{M}]^{+}$: 252.0786; found: 252.0785.

(E)-Methyl 3-[4-(coumarin-3-yl)phenyl]acrylate (9) ${ }^{1} \mathrm{H} \mathrm{NMR}\left(400 \mathrm{MHz}, \mathrm{CDCl}_{3}\right): \delta=3.82\left(3 \mathrm{H}, \mathrm{s}, \mathrm{OCH}_{3}\right), 6.49$ $(1 \mathrm{H}, \mathrm{d}, J=16.0 \mathrm{~Hz}, \mathrm{CHCHCO}), 7.32(1 \mathrm{H}, \mathrm{dd}, J=7.6,7.6$ $\mathrm{Hz}, \mathrm{H}-6), 7.38(1 \mathrm{H}, \mathrm{d}, J=8.3 \mathrm{~Hz}, \mathrm{H}-8), 7.55(1 \mathrm{H}, \mathrm{dd}$, $J=8.3,7.6 \mathrm{~Hz}, \mathrm{H}-7), 7.56(1 \mathrm{H}, \mathrm{d}, J=7.6 \mathrm{~Hz}, \mathrm{H}-5), 7.60(2$ $\left.\mathrm{H}, \mathrm{d}, J=8.1 \mathrm{~Hz}, \mathrm{H}-3^{\prime}, \mathrm{H}-5^{\prime}\right), 7.72(1 \mathrm{H}, \mathrm{d}, J=16.0 \mathrm{~Hz}$, CHCHCO), $7.76\left(2 \mathrm{H}, \mathrm{d}, J=8.4 \mathrm{~Hz}, \mathrm{H}-2^{\prime}, \mathrm{H}-6^{\prime}\right), 7.87(1 \mathrm{H}$, s, H-4). ${ }^{13} \mathrm{C} \mathrm{NMR}\left(100 \mathrm{MHz}, \mathrm{CDCl}_{3}\right): \delta=51.8\left(\mathrm{OCH}_{3}\right)$, 116.5 (C-8), 118.6 (CHCHCO), 119.5 (C-4a), 124.6 (C-6), 127.5 (C-3), 128.0 (C-5), 128.1 (C-3', C-5'), 129.0 (C-2', C6'), 131.8 (C-7), 134.8 (C-4'), 136.5 (C-1'), 140.1 (C-4), 144.0 (CHCHCO), 153.6 (C-8a), 160.3 (C-2), 167.3 (CHCHCO). MS $\left(\mathrm{EI}^{+}\right): m / z(\%)=306[\mathrm{M}]^{+}(63.15)$. HRMS $\left(\mathrm{EI}^{+}\right): \mathrm{m} / z$ calcd for $\mathrm{C}_{19} \mathrm{H}_{14} \mathrm{O}_{4}[\mathrm{M}]^{+}: 306.0892$; found: 306.0896.

(21) Chandra, V.; Srivasta, V. B. J. Indian Chem. Soc. 1969, 46, 1102.

(22) Mashraqui, S. H.; Vashi, D.; Mistry, H. D. Synth. Commun. 2004, 34, 3129.

(23) Cabri, W.; Candiani, I. Accounts Chem. Res. 1995, 28, 2.

(24) Pastre, J. C.; Correia, C. R. D. Adv. Synth. Catal. 2009, 351, 1217.

(25) Stoermer, R.; Friderici, E. Ber. Dtsch. Chem. Ges. 1908, 41, 324.

(26) Felfoldi, K.; Sutyinszky, M.; Nagy, N.; Palinko, I. Synth. Commun. 2000, 30, 1543.

(27) Yu, W. F.; Tsoi, Y. T.; Zhou, Z. Y.; Chan, A. S. C. Org. Lett. 2009, 11, 469.

(28) Stoermer, R. Ber. Dtsch. Chem. Ges. 1911, 44, 637. 